\title{
An examination of developmental assets and academic performance in higher education sport students
}

Martin Jones

University of Exeter, UK

\section{Abstract}

The purpose of this research was to explore the magnitude and direction of the relationship between developmental assets (subcategories and domain levels) and academic achievement in British students. Based on the existing theory, I hypothesised that total asset score, individual asset groups (internal and external assets), and the eight asset subcategories would have a moderate to a large positive relationship with academic achievement. 232 participants were recruited from a second-year compulsory research methods module for undergraduate sport and exercise science students at a UK based university. The participants completed the Developmental Assets Profile (DAP) at the beginning of the course and their final module grade was recorded after the course had finished. Pearson's correlation between assets and grade score revealed several significant relationships. The strongest subcategory correlation was between commitment to learning and grade score. The strongest domain level correlation was between internal assets and grade score. The current study provides evidence that developmental assets are positively associated with grade score in university students; however, the size of the relationship is smaller than previously suggested.

Keywords: thriving; positive youth development; positive psychology. 


\section{Introduction}

Thriving is a developmental concept that signifies a healthy change process linking a young person with an adult status (Lerner et al., 2003). Thriving captures a dynamic developmental process characterised by a positive positioning toward life and focus on adaptive goals (Benson and Scales, 2009). In its adjective form, thriving refers to an orientation toward life that reflects the tenets of the thriving process (Bundick et al., 2010). Benson and Scales (2009, p.85) stated that 'thriving represents the dynamic and bidirectional interplay of a young person intrinsically animated and energized by discovering his or her specialness, and the developmental contexts (people, places) that know, affirm, celebrate, encourage, and guide its expression'. Thriving encapsulates an active developmental process that may be characterised by a positive orientation toward life that is focused on adaptive goals beyond survival (Benson and Scales, 2009). Therefore, adults typically label young people as a 'thriver' if he or she is on the path to an adult status marked by making culturally valued contributions to self (for example, doing well in academia), others, and institutions (Lerner et al., 2003). Thriving is a central concept of positive youth development (i.e. the positive vision of young people as resources rather than problems). From the positive youth development perspective, thriving incorporates the absence of problem behaviours (e.g. drug abuse) and pathology (e.g. mental illness) with indicators of healthy growth (e.g. academic achievement: Scales et al., 2000). Benson (1993) first used thriving to refer to a set of 'vital signs' in adolescence. In this way, Benson advocated thriving as an outcome of positive development (i.e. a thriving orientation), rather than a process of positive development (cf. Bundick et al., 2010).

In the context of higher education, one salient indicator of thriving is the academic grade achieved by the student. Other researchers have also used academic performance as an indicator of thriving (for example, Scales et al., 2000) and have suggested that thriving occurs because of successful individual-context interaction (for example, Lerner et al. 2001). In the context of secondary education, Weissberg et al. (2003) suggested that several strategies correspond with thriving. For example, building students' socialemotional learning, providing frequent opportunities for student participation in community service, fostering caring, supportive relationships among students and teachers, and consistently rewarding positive social, health, and academic behaviour. These types of behaviour are broadly reflected in a model of youth development called the 40 developmental assets. 
The 40 developmental assets framework (Benson, 1997) is a relevant theoretical theory to study correlates of thriving in higher education because evidence consistently links high levels of assets with positive developmental outcomes, including achievement in education. Theoretically, young people who experience high levels of 'developmental assets' should achieve higher grades.

The 40 developmental assets are subcategorised into external assets (support, empowerment, boundaries and expectations, and constructive use of time) and internal assets (commitment to learning, positive values, social competencies and positive identity). Theokas et al. (2005) examined the relationship between the 40 developmental assets and thriving in adolescents by taking the specific asset subcategories and the domain level groupings of assets (internal and external) and examining whether assets contributed unique variance to a thriving index. The thriving index encompassed several thriving indicators, for instance academic success, the value of diversity, and maintenance of physical health, to mirror the assortment of components that might describe thriving. Individuals with higher scores on the thriving index had a broader selection of thriving indicators compared to people with lower overall scores. Results revealed that external assets account for approximately $28 \%$ of the variance in the thriving index and addition of internal assets contributed a further $10 \%$ to thriving. The current study extends Theokas et al.'s work by considering the relationship between the specific asset subcategories and the domain level groupings of assets and thriving in higher education. However, rather than using the thriving index, I have focused on one specific thriving indicator, namely academic success. I believe that assessing asset categories rather than broader asset domains is the most appropriate approach when predicting thriving because asset subcategories will likely provide practitioners, for instance higher education professionals, with more precise information about how to intervene to facilitate thriving. To this end, the purpose of this research was to explore the magnitude and direction of the relationship between developmental assets (subcategories and domain levels) and academic achievement in British students. Based on the existing theory, I hypothesised that total asset score, individual asset groups (internal and external assets), and the eight asset subcategories, would have a moderate to a large positive relationship with academic achievement. 


\section{Method}

\section{Participants and sampling}

Following ethical approval, I recruited 232 participants from a second-year compulsory research methods module for undergraduate sport and exercise science students at a UK based university. The purpose of the study and outcomes of the study were fully explained to the participants before they consented to take part. Feedback was not offered to students because analysis was completed after the sample had graduated from their course of study. The sample comprised 140 males (Mage $=20.53$ years, $S D=0.956$ ) and 73 females $\left(M_{\text {age }}=20.343\right.$ years, $S D=0.722$ ). This sample was chosen because the purpose of the study was to explore the magnitude and direction of the relationship between developmental assets and academic achievement in British students and these students were all British and studying at a British Higher Education Institution. I employed a prospective research design to examine whether developmental assets (collected at the beginning of the term) were associated with the final grade (collected at the end of the term following the author's university's internal and external quality assurance procedures). I administered the questionnaires in the introduction lecture (week 1) to the module and participants were told that the data would not be analysed until after the module had finished. I did not contribute to teaching or marking on the module.

\section{Measures}

\section{DAP (Developmental Assets Profile)}

The Developmental Assets Profile (DAP: The Search Institute, 2005) is a 58-item selfreport instrument to assess the adolescents' asset experiences. The DAP measures young people's reported experience of eight categories of developmental assets. The DAP comprises four external assets: support, empowerment, boundaries and expectations, and constructive use of time, and four internal assets: commitment to learning, positive values, social competencies, and positive identity. The DAP does not measure each of the 40 assets per se but rather provides an asset score, with the higher score reflected, a greater volume of assets. The DAP results based on asset categories are highly correlated with the flagship A\&B survey of individual assets, providing evidence of convergent validity (Scales, 2011). The DAP was chosen over the longer A\&B survey to reduce the burden on the participants. I adapted some of the items that were originally intended to measure school-based assets by changing school to university or teacher to lecturer in the question. 
Participants ranked items on a four-point Likert scale $(0=$ not at all or rarely, to $3=$ extremely or almost always). I calculated subscales scores by taking a mean for each asset subscale and multiplying this score by 10 to provide a score that ranged between 0 and 30. Next, I calculated an internal asset and external asset score by averaging the scale scores for the four internal asset categories and four external assets scores respectively. Lastly, I calculated a total asset score by adding together the internal and external assets scores.

\section{Grade}

The final grade represents a percentage that the student was awarded in the module. The grade assessment point for the module was a 2000 word research proposal. Several members of staff were assigned to mark the research proposals, which were then moderated by the module leader. The marks and feedback were then checked by an independent external examiner and ratified through institutional quality assurance procedures before data analysis commenced.

\section{Data analysis plan}

Given the number of tests performed $(n=11)$, I elected to set the alpha level for significance at $p \leq .005$ (1-tailed) and to calculate Bayes factors (using JASP 0.8.0.0). $\mathrm{BF}_{+0}$ quantifies the evidence for the one-sided alternative hypothesis that the population correlation is higher than zero. $\mathrm{BF}_{0+}$ quantifies the evidence for the null hypothesis relative to the one-sided alternative hypothesis that the population correlation is higher than zero. I used Jeffery's (1961) classification that Bayes factors less than one indicated no evidence, between one and three represented anecdotal evidence, between three and ten indicated moderate evidence, and Bayes factors greater than ten indicating strong evidence. I performed zero-order Pearson's (1-tailed) correlations with bias-corrected bootstrap confidence intervals based on 10,000 bootstrapped resamples to estimate the sizes of the relationships between asset subcategories, asset groups, and total assets score and grade score. 


\section{Results}

\section{Data screening and preliminary analyses}

I checked for accuracy of data input, violations of assumptions of normality, outliers, and for missing data. Little's missing completely at random (MCAR) test was not significant at the .05 level $\left(\chi^{2}=878.20, d f=909 p=.763\right)$ and missing data accounted for $0.6 \%(\leq 1 \%)$ of the total data set and was there likely MCAR, so I replaced missing data with the expectation-maximisation algorithm. I removed six mature students, as they were not from our population of interest. I also excluded 13 participants who did not submit work for summative assessment and therefore received no grade. I did not collect any follow-up data to determine why these students did not provide work. Further data screening revealed four cases that violated assumptions regarding univariate normality $(z$-score $> \pm$ 3.29) and one multivariate outlier, using a $p<.001$ criterion for Mahalanobis $D^{2}$. I removed these outliers due to the extreme scores that indicated incorrect completion of the questionnaires that is marking 1 for all items without reading the item stem. The final sample comprised 213 participants for the main analysis. I calculated estimates of internal reliability (Cronbach's $\alpha$ ) for all DAP subscales, internal asset, external assets and a total asset score. Results revealed that several of the DAP asset groups had unacceptably low internal consistency scores (<.700: Nunnally and Bernstein, 1994). Therefore it is important to note that the relationships that we reported were affected by measurement error (see Table 1, Appendix).

\section{Estimation of the relationship}

Table 1 shows the results of the eleven separate Pearson's zero-order correlations. Total asset score was positively correlated with grade score $(r=.173[.047, .296], n=213, p=$ $.006, B F_{+0}=4.071$ ). The data are 4.071 times (moderate evidence) more likely under the one-sided alternative hypothesis that the population correlation is greater than zero than the null hypothesis that the population correlation is not greater than zero. When analysing the domain level assets, the strongest correlate was internal assets $(r=.180[.049, .309]$, $n=213, p=.004, B F_{+0}=5.349$ ). The data are 5.349 times (moderate evidence) more likely under the one-sided alternative hypothesis that the population correlation is greater than zero than the null hypothesis that the population correlation is not greater than zero. Finally external assets was also positively correlated with grade score $(r=.138$ [.008, .266], $n=213, p=.022, B F_{+0}=1.256$ ). The data are 1.256 times (anecdotal evidence) 
more likely under the one-sided alternative hypothesis that the population correlation is greater than zero than the null hypothesis that the population correlation is not greater than zero. Only internal assets were statistically significant at the $p \leq .005$ (1-tailed) level.

The strongest subcategory correlation was between commitment to learning and grade score $\left(r=.193[.064, .316],. n=213, p=.002, B F_{+0}=8.871\right)$. The data are 8.871 times (moderate evidence) more likely under the one-sided alternative hypothesis that the population correlation is greater than zero than the null hypothesis that the population correlation is not greater than zero. None of the other subcategories were significant at the $p \leq .005$ (1-tailed). Only the correlation between social competencies and academic grade exceeded a Bayes factor of three but was not significant at the $p \leq .005$ (1-tailed) level ( $r=$ $\left..171[.034, .306], n=213, p=.006, B F_{+0}=3.752\right)$. Both positive values $(r=.066[-.068$, .306], $\left.n=213, p=.170, B F_{0_{+}}=4.467\right)$ and empowerment $(r=.079[-.044, .202], n=213$, $\left.p=.125, B_{0_{+}}=3.456\right)$ Bayes factors $\left(\mathrm{BF}_{0_{+}}\right)$between three and ten showed moderate evidence for the null hypothesis relative to the one-sided alternative hypothesis that the population correlation is higher than zero.

\section{Discussion}

The purpose of this research was to explore the magnitude and direction of the relationship between developmental assets (subcategories and domain levels) and academic achievement in British students. Based on the existing theory, I hypothesised that total asset score, individual asset groups (internal and external assets), and the eight asset subcategories would have a moderate to a large positive relationship with academic achievement. This article provides evidence for the contribution of developmental assets on (academic) thriving in higher education students. However, the relationship between assets and academic thriving is not as simple as previous researchers might suggest.

The relationships between total asset score and grade score, domain level asset groups and grade score, and asset subcategories and grade score, revealed mostly small relationships. This result is interesting given the magnitude of the predictive ability of developmental assets on a thriving index, which comprises several indicators of thriving. For example, Scales et al. (2000) reported that developmental assets predicted up to 54\% of the variability of a thriving index. Given that I only measured one indicator of thriving, 
specifically grade score, it is understandable that the magnitude of relationship might not be as large as previous researchers, for instance Scales et al. (2000). However, it is worth noting that the relationships are small and it is worthwhile examining why some of the subcategories were not meaningfully related to grade score.

It is not surprising that commitment to learning was significantly associated with grade score given the context of thriving in the current study. Given the claims of previous researchers that assets have a cumulative effect on thriving, it was intriguing to see that no other assets subcategories were significant (at the $p<.005$ level) and the Bayes factors revealed only anecdotal to moderate evidence, the one-sided alternative hypothesis in favor of the null hypothesis. Therefore, in the current sample, developmental assets do not appear to be as important as other researchers have suggested.

An important conclusion by Leffert et al. (1998) was that it is improbable that assets work in isolation. Leffert and colleagues (Leffert et al., 1998) stated that some asset subcategories might function as forerunners for other asset subcategories. Similarly, Theokas et al. (2005) predicted that the internal and external asset categories would interact to contribute to the prediction of thriving in adolescents. The results of the current study provide preliminary evidence that domain level developmental assets (internal and external) do not operate in isolation, and that a model of academic thriving based on the accumulation of assets may over-simplify how developmental assets contribute to academic success. Researchers and practitioners may wish to consider hypothetically relevant moderating variables, which could influence the strength and direction of the relationship between domain level asset groups, asset subcategories, and thriving.

From a practical perspective, the current findings do not support the contention that practitioners should strive to promote all assets to augment thriving. Based on the current results practitioners may wish to research ways in which they can foster a commitment to learning, for example, supportive autonomy teaching (Reeve, 2015). Based on the 95\% confidence intervals that crossed zero, practitioners should also consider the possibility that negative relationships between assets and academic success could exist and therefore they should be wary of applying a one size fits all approach to developmental assets. Specifically, some possible negative relationships (assuming that causality exists) could reveal that some asset subcategories could be inversely related to academic thriving. Before practitioners attempt to develop commitment to learning, I encourage 
replication of the present study to see whether independent researchers find similar results.

It is important to remember that the cross-sectional nature of the current study renders inferences of causality impossible. Researchers may wish to design studies that involved the manipulation of specific assets over time to examine cause and effect relationships. The current results may have also been influenced by the imprecision of measures and measurement error. For example, the DAP was originally intended for use with schoolchildren. In the current study, I changed 'school' in the questions to 'university' so that it could be used by higher education students. There could be implications and issues that influence the interpretation of results, specifically, school-based assets may be qualitatively different to higher education. Just because they are a site of education does not mean they represent the same type of developmental context for young people. Future researchers may wish to test the validity of the DAP with older participants to ensure that the measurement model is robust. The DAP does not directly measure each asset, specicifcally the DAP does not provide a score for each of the 40 assets. The DAP might not capture assets that the current participants possessed, and some unobserved assets might have been significant correlates of academic achievement. The grade recorded might not be an accurate indicator of academic thriving in the current study. Research methods are undoubtedly an important part of the science curriculum. However, some students could have thrived in more practical modules compared with the relatively abstract nature of research methods. Therefore, researchers may wish to consider an index of academic achievement that takes into consideration a range of modules across time (and possibly transcends one academic stage, for instance post-16 education results and higher education results).

\section{Conclusion}

In closing, the purpose of this research was to explore the magnitude and direction of the relationship between developmental assets (subcategories and domain levels) and academic achievement in British students. I found that internal asset domain group was correlated with grade score; however, only the commitment to learning subcategory was significantly correlated with grade score. Several 95\% Cls crossed zero which could reveal potential negative relationships, and The Bayes factors revealed that the results provided 
most anecdotal evidence for the one-sided alternative hypothesis versus the null hypothesis. Therefore, in the current sample, developmental assets do not appear to be as important as previous researchers have suggested. Practitioners and researchers may wish to replicate and expand upon these findings to establish practical ways of stimulating thriving in adolescents.

\section{References}

Benson, P.L. (1993) The troubled journey: a portrait of 6th-12th grade youth. Minneapolis, MN: The Search Institute.

Benson, P.L. (1997) All kids are our kids: what communities must do to raise caring and responsible children and adolescents. San Francisco: Jossey-Bass.

Benson, P.L. and Scales, P.C. (2009) 'The definition and preliminary measurement of thriving in adolescence', The Journal of Positive Psychology, 4(1), pp. 85-104.

Bundick, M.J., Yeager, D.S., King, P.E. and Damon, W. (2010) 'Thriving across the life span', in Lerner, R.M., Lamb, M.E., Freund, A.M. and Overton, W.F. (eds.) Handbook of life-span development, vol. 1: cognition, biology and methods. Hoboken, NJ: John Wiley and Sons, pp. 882-923.

Jeffreys, H. (1961) Theory of probability ( $3^{\text {rd }}$ edn.). Oxford: Oxford University Press.

Leffert, N., Benson, P.L., Scales, P.C., Sharma, A.R., Drake, D.R. and Blyth, D.A. (1998) 'Developmental assets: measurement and prediction of risk behaviors among adolescents', Applied Developmental Science, 2(4), pp. 209-230.

Lerner, R.M., Dowling, E.M. and Anderson, P.M. (2003) 'Positive youth development: thriving as the basis of personhood and civil society', Applied Developmental Science, 7(3), pp. 172-180. 
Lerner, R.M., Lerner, J.V., De Stefanis, I. and Apfel, A. (2001) 'Understanding developmental systems in adolescence: implications for methodological strategies, data analytic approaches, and training', Journal of Adolescent Research, 16(1), pp. 9-27.

Nunnally, J.C. and Bernstein, I. (1994) Psychometric theory. New York, NY: McGraw-Hill.

Reeve, J. (2015) 'Giving and summoning autonomy support in hierarchical relationships', Social and Personality Psychology Compass, 9(8), pp. 406-418.

Scales, P.C. (2011) 'Youth developmental assets in global perspective: results from international adaptations of the Developmental Assets Profile', Child Indicators Research, 4(4), pp. 619-645.

Scales, P.C., Benson, P.L., Leffert, N. and Blyth, D.A. (2000) 'Contribution of developmental assets to the prediction of thriving among adolescents', Applied Developmental Science, 4(1), pp. 27-46.

Theokas, C., Almerigi, J.B., Lerner, R.M., Dowling, E.M., Benson, P.L., Scales, P.C. and von Eye, A. (2005) Conceptualizing and modeling individual and ecological asset components of thriving in early adolescence', The Journal of Early Adolescence, 25(1), pp. 113-143.

The Search Institute (2005) Developmental assets profile user manual. Minneapolis, MN: Search Institute.

Weissberg, R.P., Kumpfer, K.L. and Seligman, M.E. (2003) 'Prevention that works for children and youth: an introduction, American Psychological Association, 58(6-7), p. 425-432.

\section{Author details}

Martin I. Jones is a Senior Lecturer in Sport and Exercise Psychology at the University of Exeter, UK. 


\section{Appendix}

Table 1. Descriptive statistics, correlations, internal reliability estimates and standard multiple regression of assets groups on grade percentage.

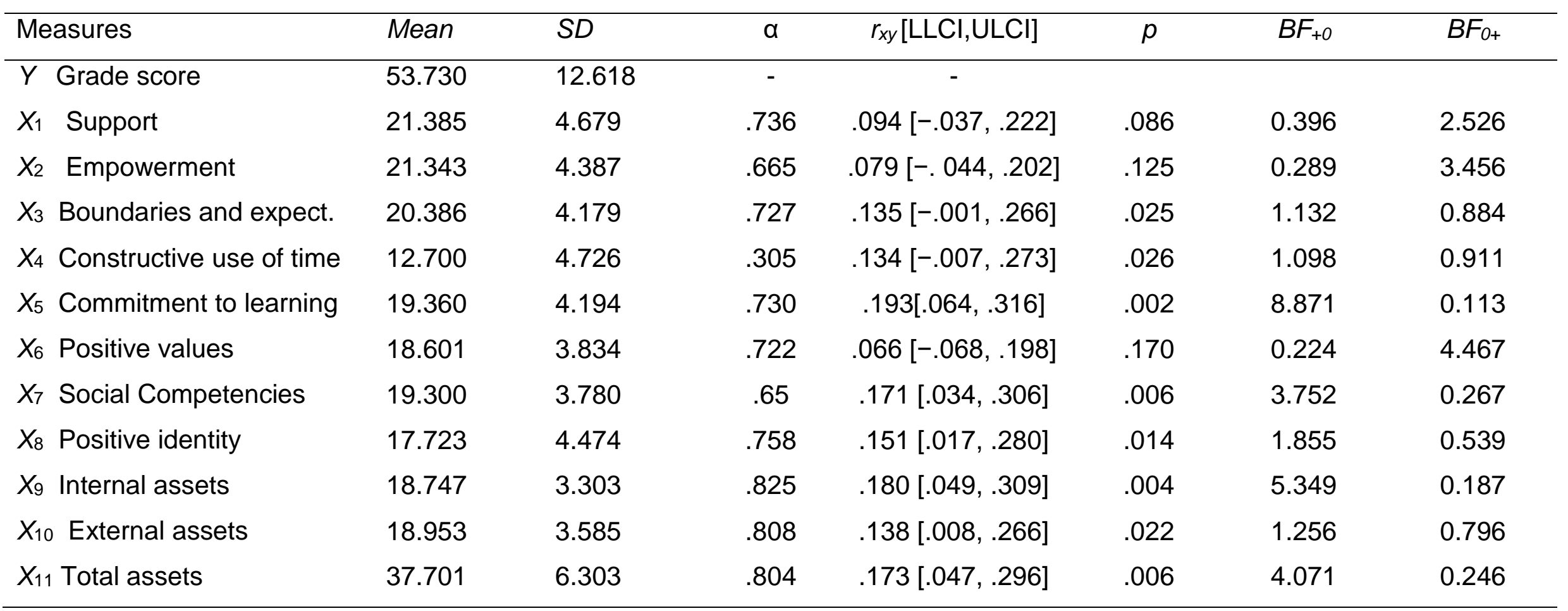

Note. Pearson's $r$ (1-tailed). BCa bootstrap Cls based on 10,000 resamples. Bayes factors based on stretch prior width of 1. 